\title{
New Developments on the Adenosine Mechanisms of the Central Effects of Caffeine and Their Implications for Neuropsychiatric Disorders
}

\author{
Sergi Ferré, MD, PhD, ${ }^{1}$ Manuel Díaz-Ríos, PhD, ${ }^{2}$ John D. Salamone, $\mathrm{PhD},{ }^{3}$ and Rui Daniel Prediger, $\mathrm{PhD}^{4}$
}

Recent studies on interactions between striatal adenosine and dopamine and one of its main targets, the adenosine $\mathrm{A}_{2 \mathrm{~A}}$ receptor-dopamine $\mathrm{D}_{2}$ receptor (A2AR-D2R) heteromer, have provided a better understanding of the mechanisms involved in the psychostimulant effects of caffeine and have brought forward new data on the mechanisms of operation of classical orthosteric ligands within $G$ protein-coupled receptor heteromers. The striatal A2AR-D2R heteromer has a tetrameric structure and forms part of a signaling complex that includes a Gs and a Gi protein and the effector adenyl cyclase (subtype AC5). Another target of caffeine, the adenosine $A_{1}$ receptor-dopamine $D_{1}$ receptor (A1R-D1R) heteromer, seems to have a very similar structure. Initially suggested to be localized in the striatum, the A1R-D1R heteromer has now been identified in the spinal motoneuron and shown to mediate the spinally generated caffeine-induced locomotion. In this study, we review the recently discovered properties of A2AR-D2R and A1R-D1R heteromers. Our studies demonstrate that these complexes are a necessary condition to sustain the canonical antagonistic interaction between a Gs-coupled receptor (A2AR or D1R) and a Gi-coupled receptor (D2R or A1R) at the adenylyl cyclase level, which constitutes a new concept in the field of G protein-coupled receptor physiology and pharmacology. A2AR antagonists targeting the striatal A2AR-D2R heteromer are already being considered as therapeutic agents in Parkinson's disease. In this study, we review the preclinical evidence that indicates that caffeine and A2AR antagonists could be used to treat the motivational symptoms of depression and attention-deficit/hyperactivity disorder, while A1R antagonists selectively targeting the spinal A1R-D1R heteromer could be used in the recovery of spinal cord injury.

Keywords: caffeine, adenosine receptors, dopamine receptors, GPCR heteromers, striatum, spinal cord, spinal cord injury, depression, attention-deficit/hyperactivity disorder

\section{Introduction}

T HE PSYCHOSTIMULANT EFFECTS of caffeine have been classically related to its ability to act as a nonselective antagonist of adenosine receptors. More specifically, its psychomotor stimulant-reinforcing effects have been mostly ascribed to its ability to block striatal adenosine $A_{2 A}$ receptors (A2AR), whereas its arousing effects have been mostly associated with blockade of $A_{1}$ receptors (A1R) that mediate the homeostatic sleep control of adenosine. ${ }^{1,2}$ Nevertheless, this is an oversimplification, since A1R are also involved in the psychomotor effects of caffeine and A2AR are also involved in the adenosine-mediated sleep control. ${ }^{1,2}$ Experimental data

\footnotetext{
${ }^{1}$ Integrative Neurobiology Section, National Institute on Drug Abuse, Intramural Research Program, National Institutes of Health, Baltimore, Maryland.

${ }^{2}$ Department of Anatomy and Neurobiology, Institute of Neurobiology, University of Puerto Rico, Medical Sciences Campus, San Juan, Puerto Rico.

${ }^{3}$ Department of Psychological Sciences, University of Connecticut, Storrs, Connecticut.

${ }^{4}$ Department of Pharmacology, Federal University of Santa Catarina, Florianópolis, Brazil.

This manuscript is part of a series of reviews from symposia on caffeine and adenosine presented in the meeting "Purines 2018 International," in Foz do Iguaçu, Brazil (June 19-22, 2018).

(c) Sergi Ferré, et al. 2018; Published by Mary Ann Liebert, Inc. This Open Access article is distributed under the terms of the Creative Commons Attribution Noncommercial License (http://creativecommons.org/licenses/by-nc/4.0/) which permits any noncommercial use, distribution, and reproduction in any medium, provided the original author(s) and the source are cited.
} 
accumulated during the last two decades have substantiated that striatal A2AR form heteromers with dopamine $D_{2}$ receptors (D2R), which are specifically localized in one of the two striatal efferent neurons, the striatopallidal neuron. ${ }^{3}$ Although the initial interpretation of the mechanisms by which caffeine influenced striatal A2AR signaling was simply a competitive inhibition of adenosine binding to the A2AR, recent studies indicate a dependence on an unexpected allosteric interaction within the A2AR-D2R heteromer. ${ }^{4}$

Those studies also suggested a specific quaternary structure of the heteromer, an A2AR-D2R heterotetramer, a concept that was very recently confirmed and expanded to the existence of a precoupled complex that also includes the effector, adenylyl cyclase subtype 5 (AC5). ${ }^{5}$ As described below, an important conceptual finding from this study was the demonstration that this macromolecular complex provides the necessary framework for the canonical Gi-Gs interaction at the AC level, sustaining the ability of a Gi-coupled receptor (D2R) to counteract AC activation mediated by a Gs-coupled receptor (A2AR). ${ }^{5}$ It was also initially assumed that two subpopulations of striatal A2AR, forming and not forming heteromers with $\mathrm{D} 2 \mathrm{R}$, were necessary to explain some pharmacological findings. ${ }^{6}$ Recent studies allowed revisiting this hypothesis and readdress the biochemical and behavioral effects of A2AR ligands in the frame of one main population of striatal A2AR, the A2AR-D2R heterotetramer-AC5 complex, localized in the striatopallidal neuron., ${ }^{3,7}$

However, caffeine does not only bind to the striatal A2AR-D2R-AC5 complex, but also to the Gi-coupled A1R localized in the striatonigral neuron, which form heteromers with the Gs-coupled dopamine $\mathrm{D}_{1}$ receptor $(\mathrm{D} 1 \mathrm{R})^{8}$ and, also, caffeine binds to A1R-A2AR heteromers localized in the corticostriatal glutamatergic terminals. ${ }^{9}$ Therefore, these additional striatal targets should also contribute to the pharmacological effects of caffeine. Interestingly, the A1R-D1R has been recently found in another region of the CNS, in the spinal cord, where it plays an important role in the modulation of the intrinsic excitability of the spinal motoneuron $(\mathrm{MN}){ }^{10}$ Significantly, as reviewed in this study, the A1R-D1R heteromer plays a major role in the spinally generated locomotion induced by caffeine. ${ }^{10,11}$

Initial translational studies on the possible therapeutic effects of caffeine and selective A2AR antagonists targeting striatal $\mathrm{A} 2 \mathrm{AR}$ were oriented to the treatment of akinesia in Parkinson's disease. ${ }^{12,13}$ Those studies implicitly considered A2AR-D2R heteromers localized in the dorsal striatum, whereas a large number of studies also demonstrate the very same presence of A2AR and $\mathrm{D} 2 \mathrm{R}$ in the nucleus accumbens, in the ventral striatopallidal neuron, implicating A2AR-D2R heteromers in more motivational aspects of the basal ganglia., ${ }^{3,14,15}$ As reviewed in this study, with this additional target, the possible clinical application of caffeine and A2AR antagonists are extending to "apathy", or "anergia", a major negative symptom of several neuropsychiatric disorders, including depression, schizophrenia, substance use disorders, and also Parkinson's disease. ${ }^{3,14,15}$ Finally, recent experimental results obtained in spontaneously hypertensive rats (SHR), a validated model of attentiondeficit/hyperactivity disorder (ADHD), made us revisit the possible therapeutic role of caffeine and A2AR antagonists for the treatment of this disorder. ${ }^{16}$

\section{Molecular Mechanisms of the Psychostimulant Effects of Caffeine: The Striatal A2AR-D2R Heterotetramer-AC5 Complex}

Recent studies on interactions between striatal adenosine and dopamine and one of its main targets, the A2AR-D2R heteromer, have not only provided a better understanding of the mechanisms involved in the psychostimulant effects of caffeine, but they have also brought new and even counterintuitive data on the mechanisms of operation of classical orthosteric ligands within $G$ protein-coupled receptor (GPCR) heteromers. First, there is the demonstration of the heterotetrameric structure of the A2AR-D2R heteromer using several biophysical and antibody-based techniques in mammalian transfected cells and striatal cells in culture. ${ }^{4,5}$ The A2AR-D2R heteromer is composed of A2AR and D2R homodimers with the four receptor units (protomers) forming a linear arrangement; the two internal A2AR and D2R protomers form the heteromeric interface and the two external protomers couple to the $\alpha$ subunit of their respective cognate $\mathrm{G}$ proteins (one $\mathrm{G}$ protein per homodimer), Gs (more specifically Golf) for the A2AR and Gi for the D2R. ${ }^{5}$ This arrangement allows the precoupling of the heterotetramer with the effector AC5 and, as mentioned above, provides the framework for the antagonistic interaction of $\mathrm{A} 2 \mathrm{AR}$ and $\mathrm{D} 2 \mathrm{R}$ agonists at the $\mathrm{AC}$ level (Fig. 1). ${ }^{5}$

The topology of AC5 includes a long N-terminal domain (NT), which is known to bind to $\beta \gamma$ subunits of $\mathrm{G}$ proteins, ${ }^{17}$ two large quasi-identical cytoplasmic catalytic domains ( $\mathrm{C} 1$ and $\mathrm{C} 2$ ), which bind to each other and form the catalytic core at their interface, and two membrane-spanning domains, M1 and M2, each with six transmembrane domains (TMs). ${ }^{18}$ The Gs $\alpha$ subunit can bind to $\mathrm{C} 2$, which increases the affinity between $\mathrm{C} 1$ and $\mathrm{C} 2$, promoting catalysis, whereas $\mathrm{Gi} \alpha$ binds to $\mathrm{C} 1$ and has the opposite effect. ${ }^{18}$ The nearly symmetrical arrangement of $\mathrm{C} 1$ and $\mathrm{C} 2$ allows the simultaneous binding of Gs $\alpha$ and Gi $\alpha,{ }^{19}$ and, therefore, the canonical antagonistic interaction. The use of synthetic peptides with the amino acid sequence of all the TMs of the A2AR, D2R, and AC5, with their ability to interfere with their specific intermolecular interactions (interfaces), provided a fundamental tool to demonstrate, first, the quaternary structure of the A2AR-D2R heterotetramer-AC5 complex in the inactive state. We were able to demonstrate, for the 


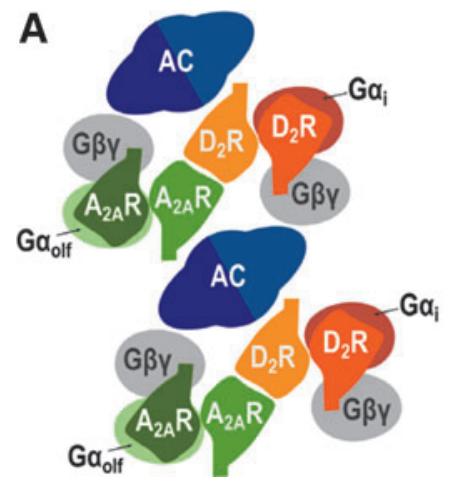

B

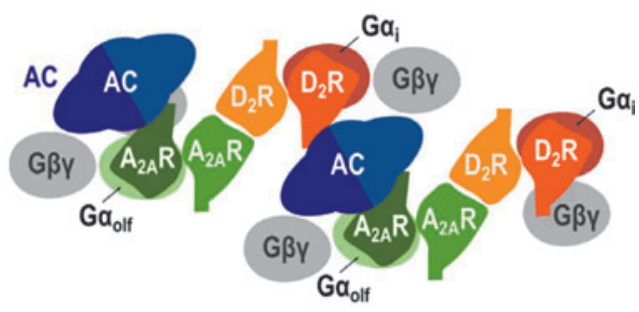

FIG. 1. The A2AR-D2R

heterotetramer-AC5 complex. Schematic slice-representation, viewed from the extracellular side, of the minimal functional unit of the A2AR-D2R heterotetramer in complex with Gs (more specifically Golf) and Gi proteins and adenyl cyclase (subtype AC5), in the absence (A) and presence (B) of $\mathrm{A} 2 \mathrm{AR}$ and $\mathrm{D} 2 \mathrm{R}$ agonists, which induce a rearrangement of the heterotetramer-AC5 interfaces (modified from Ferré et $a .^{3}$ ). A2AR-D2R, adenosine $\mathrm{A}_{2 \mathrm{~A}}$ receptor-dopamine $\mathrm{D}_{2}$ receptor. first time, a functional role of the AC5 TMs: their ability to establish intermolecular interactions with GPCR TMs. ${ }^{5}$

Computational modeling indicated that, to allow the canonical interaction, a minimal functional A2ARD2R-AC5 complex should include at least two molecules of heterotetramers alternating with two molecules of AC5 (Fig. 1A). In this way, in the inactive state of the A2AR-D2R heterotetramer-AC5 complex, AC5 establishes direct TM interactions with one receptor and indirect intracellular interactions with the other receptor through the NT coupling to $\beta \gamma$ subunits. Second, we could also demonstrate the ligand-induced dynamic changes of this quaternary structure. Basically, the agonist does not induce any change in the homodimeric and heterodimeric interfaces, leaving intact the quaternary structure of the A2AR-D2R heterotetramer. ${ }^{5}$ On the other hand, ligands induce a significant rearrangement of the interfaces of the receptors with AC5, which allows the simultaneous interaction of the $\alpha$ subunits of Gs and Gi with the corresponding catalytic domains of AC5 (Fig. 1B). ${ }^{5}$ The rearrangement fits with the well-established ligand-induced relative movement of $\beta \gamma$ from the $\alpha$ subunits of the $\mathrm{G}$ protein. Thus, the $\beta \gamma$ subunits normally occlude the site of interaction of the $\alpha$ subunit with the corresponding catalytic domain of $\mathrm{AC} 5,{ }^{20}$ and the movement of $\beta \gamma$ releases this site of interaction and simultaneously pulls and approximates AC5 to the $\alpha$ subunit. $^{5}$

At a more general level, these advances in our knowledge of the dynamic molecular structure of the A2AR-D2R heterotetramer represent a "promotion" of the functional significance of receptor heteromers. Interactions between receptors at the level of AC were classically assumed to belong to downstream interactions, also called interactions "at the second-messenger level." These results imply that the canonical Gi-Gs interaction at the AC level is a functional property of a GPCR heteromer. In fact, TM peptides that specifically destabilize A2AR-D2R heteromerization completely abrogated the canonical interaction, the ability of D2R agonists to antagonize A2AR-mediated AC activation. ${ }^{5}$
Another groundbreaking pharmacological finding in the framework of the A2AR-D2R heteromer has been realizing that the ability of caffeine and A2AR antagonists to counteract some biochemical and behavioral effects of adenosine or, more precisely, A2AR agonists does not depend on a classical competition for the same orthosteric site in the A2AR. Surprisingly, the counteraction depends on the simultaneous occupancy of the two orthosteric sites of the A2AR dimer of the A2AR-D2R heterotetramer. ${ }^{4}$ When performing radioligand-binding control experiments, we found that $\mathrm{A} 2 \mathrm{AR}$ antagonists, including caffeine, have the same allosteric properties than A2AR agonists on D2R agonist binding. The same as initially found for A2AR agonists, ${ }^{21}$ caffeine and A2AR antagonists displaced the binding of a tritiated D2R agonist from membrane preparations of mammalian transfected cells or mammalian striatum, decreasing its affinity. ${ }^{4}$ This would mean that any A2AR ligand, agonist, or antagonist, induces a negative allosteric modulation of $\mathrm{D} 2 \mathrm{R}$ agonists in the A2AR-D2R heteromer.

But this interpretation represented a challenge to our previously formulated hypothesis of the key involvement of the negative allosteric modulation between A2AR and $\mathrm{D} 2 \mathrm{R}$ agonists in the motor depressant effects of A2AR agonists and the motor-activating effects of caffeine and A2AR antagonists. ${ }^{22}$ We should then logically expect A2AR agonists and antagonists to show opposite biochemical effects. In fact, in patch-clamp experiments with identified striatopallidal neurons, we had showed that an A2AR antagonist blocks the ability of an A2AR agonist to counteract a D2R agonist-induced inhibition of neuronal excitability. ${ }^{23}$ Nevertheless, we obtained an encouraging unexpected finding: when coapplied, A2AR agonists and antagonists counteracted each other's effects. With low concentrations, caffeine and A2AR antagonists counteracted the effect of an A2AR agonist on D2R agonist binding, but with high concentrations, they also caused a significant decrease in D2R agonist binding. ${ }^{4}$

These experimental results would completely agree with the presence of two molecularly interconnected molecules 
of A2AR, an A2AR homodimer, in the A2AR-D2R heteromer, which would sustain a negative homomeric allosteric modulation between an orthosteric A2AR agonist and an orthosteric A2AR antagonist. Occupation of the A2AR homodimer with either an agonist or an antagonist induces the negative heteromeric allosteric modulation of the D2R agonist, reduces its binding and efficacy. But combined occupation of the A2AR homodimer with an agonist and an antagonist, because of a negative homomeric allosteric modulation between both A2AR ligands, prevents the heteromeric allosteric modulation.

The negative homomeric allosteric modulation between A2AR agonists and antagonists, and therefore dimeric nature of the $\mathrm{A} 2 \mathrm{AR}$, was confirmed with dissociation experiments with a tritiated A2AR antagonist in striatal membrane preparations. An A2AR agonist, but not caffeine or an A2AR antagonist, significantly modified the dissociation rate of the labeled antagonist, indicating the presence of a specific allosteric interaction between the A2AR agonist and the tritiated A2AR antagonist. ${ }^{4}$ We could also demonstrate the functional significance of the negative homomeric allosteric modulation using the same experimental patch-clamp approach previously used to demonstrate the negative heteromeric allosteric modulation between the A2AR and D2R agonists in the striatopallidal neuron. ${ }^{23}$ Thus, the same A2AR antagonist that counteracted the effect of the A2AR agonist, when applied alone, also counteracted a D2R agonist-induced inhibition of the striatopallidal neuron excitability. ${ }^{4}$

Our results therefore indicate that caffeine and selective A2AR antagonists produce psychostimulant effects, not just by competing with adenosine for its binding to the A2AR, but by exerting a negative allosteric modulation within the A2AR homomer of the A2AR-D2R heterotetramer. Under normal conditions, by means of the negative heteromeric allosteric modulation, endogenous adenosine tonically inhibits psychomotor activation mediated by a tonic activation of D2R by endogenous dopamine. Caffeine, by means of the negative homomeric allosteric modulation, counteracts the effect of adenosine and produces psychomotor activation.

It is well known that high doses of caffeine produce psychomotor depression. According to our model, this is due to the ability of caffeine to completely displace endogenous adenosine and induce the negative heteromeric allosteric modulation of endogenous dopamine binding to the D2R. We could demonstrate our hypothesis with experiments of locomotor activity in rats upon coadministration of the A2AR agonist CGS 2180 and an A2AR antagonist, KW-6002 (istradefylline), used in Parkinson's disease. ${ }^{13}$ Low doses of KW-6002 produced locomotor activation, whereas high doses produced locomotor depression. Significantly, as we anticipated, a dose of CGS 21680 that also produced locomotor depression when administered alone counteracted the locomotor depression of the high locomotor-depressant dose of KW-6002. ${ }^{4}$

\section{Caffeine-Induced Spinally Generated Locomotion: The Spinal A1R-D1R Heteromer}

Adenosine also acts as an intrinsic modulator suggested to have an important role in spinally generated rhythmic movements. ${ }^{24}$ It has been shown that glialderived adenosine can modulate spinal motor networks in mice by facilitating inhibitory neurotransmission. ${ }^{25}$ In this study, the application of adenosine led to a reduction in the frequency of locomotor activity recorded from ventral roots. On the other hand, the application of the non-selective adenosine receptor antagonist theophylline or a selective A1R antagonist, but not a selective A2AR antagonist, caused an increase in locomotor burst frequency, demonstrating that endogenously derived adenosine activates A1Rs during locomotor network activity. Since the effect of theophylline was counteracted by glial toxins, this study indicated that endogenous purinergic glial signaling (through activation of A1Rs) is important for the intrinsic modulation of cycle frequency of locomotor activity in mammals. ${ }^{25}$

The same research group studied the effects of the application of adenosine to interneurons (INs) and MNs of the mouse lumbar cord. ${ }^{26}$ By acting primarily through A1R, adenosine hyperpolarized INs and reduced the frequency and amplitude of their synaptic inputs. ${ }^{26}$ On the other hand, adenosine application produced a net depolarizing effect on MNs while reducing the frequency and amplitude of their synaptic inputs. ${ }^{26}$ These results suggested differential A1R-mediated effects of adenosine in the mouse spinal cord, with a general suppressing action on ventral horn INs and potentially maintaining MN excitability. It was suggested that these differential effects in INs and MNs may allow for adaptation of the locomotor pattern generated by interneuronal networks while helping to ensure the maintenance of overall motor output. ${ }^{25,26}$

More recently we performed experiments with caffeine and selective A1R ligands to understand the specific mechanisms of A1R modulation on the spinal locomotor central pattern generator (CPG). Addition of caffeine or an A1R antagonist onto an ongoing fictive locomotor rhythm significantly increased cycle frequency while reducing burst duration of the MN output. This suggested that the A1R is the primary target of caffeine's spinal modulatory effects. ${ }^{11}$ Also, caffeine and an A1R antagonist failed to stimulate ongoing locomotor activity in the absence of dopamine or in the presence of a D1R antagonist, supporting an A1R-D1R-dependent mechanism of action. ${ }^{11}$ Caffeine and the selective A1R antagonist failed to stimulate an ongoing locomotor rhythm in the presence of an inhibitor of cAMP-dependent protein kinase A (PKA). Altogether, these results suggested a stimulant effect of caffeine on the lumbar CPG controlling hindlimb locomotion through the inhibition of A1Rs and subsequent activation of D1R through a PKA-dependent intracellular mechanism. ${ }^{11}$ 
This study led to our most recent experiments, which demonstrated that heteromerization of the Gi-coupled A1R with the Gs-coupled D1R was occurring in spinal MNs of neonatal mice. ${ }^{10}$ The cellular location of these potential receptor heteromers was first confirmed using antibodies directed toward A1R and D1R in postnatal 0-5 (P0-P5) mouse lumbar spinal cord slices, localization of the network controlling hindlimb locomotion. ${ }^{27-30}$ Overlay of the A1R and D1R immunostaining confirmed that colabeling of both receptors was mostly found within lumbar MNs ${ }^{10}$ which were visually identified by their location, size, and clustering.

Previous studies had already established that $\mathrm{D}_{1}$-like receptors (D1R and D5R) and $\mathrm{D}_{2}$-like receptors (D2R, D3R, and $\mathrm{D} 4 \mathrm{R}$ ) are mostly segregated in the spinal cord and that they play opposite modulatory effects on spinal CPG of locomotion. ${ }^{31,32}$ Although there are differences among species, in the mouse, $\mathrm{D}_{2}$-like receptors are highly expressed in laminae I-II of the dorsal horn, where they mediate inhibitory effects. On the other hand, $\mathrm{D}_{1}$-like receptors, are highly expressed in the ventral horn, in lamina IX, including the MNs, where they mediate excitatory effects. ${ }^{31-33}$

With a series of parallel experiments using biophysical and antibody-based techniques in mammalian transfected cells, we could demonstrate that A1R and D1R form heteromers with their respective cognate $G$ proteins, supporting very similar structural and functional properties of that of the A2AR-D2R heteromers. ${ }^{10}$ In fact, these experiments demonstrated an interaction at the AC level, since the ability of Gi-coupled A1R to counteract Gs-coupled D1R-mediated AC activation could be counteracted by a TM peptide that destabilized A1R-D1R heteromer interface (with the amino acid sequence of TM 5, but not TM 7 of the D1R; TM5 and TM7 peptides, respectively). ${ }^{10}$

Additional evidence for A1R-D1R heteromer formation in transfected cells involving TM 5 of the D1R was provided by using the proximity ligation assay (PLA). This technique permitted the direct detection of molecular interactions between endogenous (nontransfected) A1R and D1R. The expression of A1R-D1R heteromers in the lumbar spinal cord was demonstrated with PLA in slices from neonatal mouse spinal cords (lumbar region) from P4, P6, and P11 animals. ${ }^{10}$ A1R-D1R complexes were selectively detected irrespective of the postnatal period in cells from lamina IX, corresponding to MNs. Importantly, pretreatment of the spinal cord slices with TM5, but not with TM7, significantly decreased PLA staining, therefore identifying A1R-D1R heteromers in mouse spinal MNs. ${ }^{10}$

After establishing the anatomical localization of A1R-D1R heteromers in the spinal MN, their functional and pharmacological significance was confirmed by intracellular electrophysiological patch-clamp recordings. The application of caffeine or an A1R antag- onist, in the presence of either dopamine or a D1R agonist, increased the intrinsic excitability of spinal MNs but not INs. These results could be explained by the ability of caffeine or the A1R antagonist to potentiate the depolarizing effects of dopamine within the A1R-D1R heteromers of the spinal MN. ${ }^{10}$ This was demonstrated in experiments using the disruptive peptides. In these experiments, the application of caffeine and the D1R agonist did not increase the intrinsic excitability of lumbar MNs if applied in the presence of TM5 but not TM7. ${ }^{10}$ This study demonstrates that A1R-D1R heteromers play a significant role in the control of the excitability of spinal MNs. More specifically, A1RD1R localized in the spinal MNs mediate the ability of adenosine to exert a tonic inhibitory modulation of a D1R-dependent, dopamine-mediated increase in neuronal excitability.

A1R-mediated effects of adenosine have not only been shown to modulate the mouse spinal locomotor network, ${ }^{11,24-26}$ but also to suppress neuronal cell death induced by ischemia in rat spinal MNs. ${ }^{34}$ This would imply that A1R agonist would improve, while A1R antagonists and caffeine would worsen inflammation-mediated degeneration in spinal cord injury (SCI) and other spinal cord inflammation-dependent conditions. In fact, caffeine has been shown to aggravate the secondary inflammatory-dependent degeneration of experimental SCI. ${ }^{35}$ On the other hand, in vivo studies with dopamine agonists and antagonists on hindlimb movement generation in mice with a completely transected spinal cord transected at the low thoracic level found that the application of D1R, but not D2R, agonists acutely elicits rhythmic locomotor-like movements and nonlocomotor movements in untrained and nonsensory stimulated animals. ${ }^{36}$

Therefore, spinal dopaminergic neurotransmission, and more specifically D1R, are being considered as potential new targets to promote recovery of locomotor function following $\mathrm{SCI}^{32}$ Our studies indicate that, even more specifically, the MN A1R-D1R heteromer, should be addressed as a main target and that caffeine and A1R antagonists, alone or in combination with D1R agonists, could be beneficial during noninflammatory stages of SCI. An important factor to be considered within the framework of GPCR heteromers is their ability to modify ligand properties, as compared with the same GPCRs when not forming heteromers. ${ }^{37}$ The proof of concept came from the demonstration of a very significant different affinity of an A2AR antagonist (SCH442416) when coexpressed with the A2AR alone or with the A2AR, both in mammalian transfected cells or in the rodent striatum (comparing wild-type with conditional striatal A2AR knockout mice). ${ }^{3,6}$ Therefore, A1R antagonists with preferential affinity for the A1R-D1R heteromers could be useful therapeutically to treat the deleterious effects of a SCI. 
Caffeine and Selective A2AR Antagonists as New Therapeutic Tools for the Motivational Symptoms of Depression

Although depression is typically thought of as involving emotional symptoms, such as sadness and negative affect, it also is important to emphasize that depression is characterized by motivational dysfunctions such as apathy or anergia, psychomotor retardation, reduced exertion of effort, fatigue, and a general lack of behavioral activation. ${ }^{38}$ These motivational symptoms of depression are highly debilitating, and they are very difficult to treat. For example, although serotonin-selective uptake inhibitors (SSRIs) are the most commonly used drug treatments for depression, and are very useful for treating anxiety and mood-related symptoms, they are relatively poor at treating motivational dysfunction. ${ }^{39}$ Moreover, motivational dysfunctions in human psychopathology are not limited to depression; people with schizophrenia and Parkinson's disease also show negative symptoms, anergia, and fatigue. For all these reasons, it is important to develop animal models of activational or effort-related psychiatric symptoms.

In the last few years, studies of effort-related choice behavior have been developed as formal models of the motivational symptoms of depression. Effort-based choice tasks allow organisms to choose between a more preferred reinforcer that can only be obtained through a high degree of effort, versus a low effort/less valued option. In rodents, the high effort activities include things like lever pressing on operant schedules, climbing a barrier, or running in a running wheel. Considerable evidence indicates that conditions associated with motivational dysfunction in humans, including some specifically associated with depression, produce a low-effort bias (i.e., decreased selection of the higheffort option), although these same conditions do not affect food intake or preference between the different reinforcers in free-feeding tests. For example, dopamine antagonists, such as haloperidol, and conditions associated with depression in humans, such as stress, or administration of the vesicular monoamine transport inhibitor tetrabenazine, alter choice behavior by decreasing selection of the high-effort option and increasing selection of the low-effort option. ${ }^{38,40-49}$

In addition, consistent with studies showing that there are inflammation-related aspects of depressive symptomatology, administration of the proinflammatory cytokines, interleukin (IL) $1 \beta$ and IL-6, have been shown to induce a low-effort bias. ${ }^{50,51}$ The use of effort-related choice tasks in rodents as a model of depressive symptoms is validated by research reporting that people with major depressive disorder also show a low-effort bias in human tests of effort-based choice ${ }^{52,53}$ Drug development studies have found that the low-effort bias induced by tetrabenazine is not reversed by the SSRIs fluoxetine and citalopram, but are reversed by drugs that block catecholamine uptake, or specifically block DA transport. ${ }^{41,43,45,46}$

Considerable research in this area also has focused on the effects of drugs that act on adenosine. Administration of the adenosine A2AR agonist CGS 2168, directly into nucleus accumbens, produced a low-effort bias, and reduced exertion of effort on lever pressing tasks. ${ }^{53,54}$ Furthermore, A2AR antagonists such as istradefylline, MSX-3, MSX-4, and preladenant are able to reverse the low-effort bias induced by the D2R antagonists, haloperidol and eticlopride, ${ }^{55-59}$ and the vesicular storage antagonist tetrabenazine. ${ }^{41,43,49}$ In contrast to these effects of A2AR antagonism, blockade of A1R does not reverse the effects of DA antagonism at either D1R or $\mathrm{D} 2 \mathrm{R} .^{40,57,60}$ The greatest reversal effects are seen when an A2AR antagonist is used to reverse the effects of a D2R antagonist, ${ }^{40,57,58}$ which is consistent with anatomical data on the extensive colocalization of these two receptors, and their molecular interactions in the striatum.

Moreover, studies of postsynaptic metabotropic signaling cascades demonstrate that A2AR antagonists are able to reverse the expression of $c$-fos and phosphorylation of DARPP-32 (Thr34) by D2R antagonists and tetrabenazine. ${ }^{41,59,60}$ The latter studies provide a cellular marker of the A2AR-D2R interaction that is occurring at behaviorally relevant doses of drugs that act on these receptors. A2AR antagonism also reverses the low-effort bias induced by proinflammatory cytokines. ${ }^{50,51}$ These observations in animal models are consistent with human clinical studies reporting that the A2AR antagonist istradefylline can also improve fatigue symptoms in Parkinson's disease patients. ${ }^{61,62}$

The nonselective adenosine antagonists, caffeine and theophylline, also have been shown to exert effects on tests of effort-based choice. ${ }^{15}$ Caffeine and theophylline reversed the effects of D2R antagonism on effort-related choice ${ }^{56,60}$ In rats that were not food restricted, caffeine ( 5 or $10 \mathrm{mg} / \mathrm{kg}$ ) increased lever pressing output in rats tested on a progressive ratio/chow feeding choice task, but only among low responders. ${ }^{63}$ Taken together, these studies suggest that drugs acting on A2A receptors, as well as nonselective adenosine antagonists, may have utility for treating motivational dysfunction in depressed people. ${ }^{15}$

\section{Caffeine and Selective A2AR Antagonists as Therapeutic Tools for the Cognitive and Emotional Impairments in ADHD}

ADHD is one of the most common chronic childhoodonset psychiatric disorders, with an estimated global prevalence of $5.9-7.1 \%$ among children/adolescents and $1.2-7.3 \%$ among adults. ${ }^{64,65}$ ADHD persists in many cases into adulthood, whose occurrence is associated with attention deficits, hyperactivity, and/or cognitive impulsiveness. A primary role of disturbances in 
frontocortical dopaminergic neurotransmission in ADHD is the basis for the current pharmacological treatment with psychostimulants, more commonly methylphenidate. $^{66,67}$ However, the understanding of the modulatory influence of adenosine receptors on dopamine neurotransmission has increased in recent years. Thus, there is sufficient evidence that the adenosine receptors represent a target for the development of drugs for the treatment of diverse disorders associated with the dysregulation in dopamine neurotransmission, including ADHD. ${ }^{68,69}$

Although beneficial effects of caffeine on ADHD symptoms were first described by Schnackenberg in $1973,{ }^{70}$ the clinical use of caffeine was poorly investigated over the last four decades. Some authors demonstrated an improvement of ADHD symptoms with caffeine, ${ }^{71-74}$ whereas others have not found convincing positive effects. ${ }^{75-78}$ These apparent discrepancies are most probably attributed to methodological differences. For this reason, the general view in the field is that adjunctive caffeine is not contraindicated for the treatment of ADHD, but it might not be a viable replacement for the firstline treatment for ADHD, such as methylphenidate. ${ }^{79}$

In a meta-analysis study, Leon reported that the small number of studies, the small sample size (14.4 subjects/ study), and the great variation in caffeine doses are the main limiting factors and should be considered with caution. ${ }^{80}$ Moreover, there is no consensus in the literature about the optimal caffeine dose to elicit benefits on ADHD symptoms. Some researchers reported that low caffeine doses are more effective in attenuating the behavioral impairments in ADHD, contrasting with the earliest description by Schnackenberg, ${ }^{70}$ in which the benefits of caffeine were observed in doses higher than $200 \mathrm{mg}$ daily. This inconsistency and lack of agreement on the "optimal" dose of caffeine could be related to the general inability to monitor the $\mathrm{A} 2 \mathrm{AR}$ gene polymorphisms that moderate the intake of coffee and caffeine. ${ }^{81}$

On the other hand, many studies have shown that caffeine treatment improved memory and attention deficits and also normalized dopaminergic function in adolescent and adult SHR, a validated animal model of ADHD. Acute treatment with caffeine improved the social memory, ${ }^{82}$ object recognition memory, ${ }^{83}$ and spatial learning deficits $^{84}$ exhibited by adult SHR in different behavioral paradigms. An improvement on short-term social memory was also achieved after an acute administration of the selective A2AR antagonist, but not with a selective A1R antagonist. ${ }^{82}$

Of high importance, chronic caffeine treatment provided long-term cognitive benefits in SHR. Chronic caffeine administration during adolescence was able to improve short-term recognition ability in adult SHR, ${ }^{85}$ improved memory and attention deficits, and also normalized dopaminergic function by reducing dopamine reuptake in the striatum and frontal cortex of SHR. ${ }^{86}$ More recently, Nunes et al. compared the effects of free caffeine intake from childhood to adolescence in behavior and in brain-derived neurotrophic factor (BDNF) signaling pathway in male and female SHR. ${ }^{87}$ The authors observed that caffeine intake improved recognition memory impairments in both genders. However, spatial memory was recovered only in female SHR. Additionally, caffeine normalized BDNF in males and truncated TrkB receptor in both sexes. ${ }^{87}$ These results would comply with the well-demonstrated interactions between A2AR-mediated adenosine and TrkB-mediated BDNF signaling cascades. ${ }^{88}$

Finally, we recently investigated the effects of chronic caffeine intake and physical exercise on the emotional impairments observed in SHR. ${ }^{16}$ Male SHR were submitted, from adolescence (30 days old) to adulthood, to the association of caffeine intake $(0.3 \mathrm{mg} / \mathrm{mL}$ in drinking water) with and without voluntary physical exercise in running wells for 6 weeks. After that, depressive- and anhedonic-like behaviors were evaluated in the forced swimming and splash tests. The results indicate significant and independent antidepressant-like effects of chronic caffeine intake and physical exercise in SHR, without alterations of anhedonic-like behaviors. These findings provide the first evidence of beneficial effects of chronic caffeine intake on emotional impairments observed in an animal model of ADHD. Altogether, the review of clinical and preclinical studies of caffeine and ADHD strongly suggest that we might need to readdress the possible therapeutic role of caffeine in ADHD and better controlled studies (also including control of A2AR gene polymorphisms) need to be performed.

\section{Concluding Remarks}

GPCR heteromer is currently defined as a macromolecular complex composed of at least two different receptor units (protomers) with biochemical properties that are demonstrably different from those of its individual components. ${ }^{89}$ GPCR heteromers can provide unique properties to specific ligands, which provide new avenues for drug development. ${ }^{37}$ Recently, we have been particularly interested in GPCR heteromers with one of the protomers coupled to a Gs protein and the other to a Gi protein. Our working hypothesis has been that these heteromers have a tetrameric structure, forming a GPCR heterotetramer with two homodimers, each one coupled to its preferred G protein. ${ }^{90}$ This structure would then provide the framework for the canonical interaction between a Gs and a Gi-coupled receptor at the AC level, the ability of a Gicoupled receptor to counteract $\mathrm{AC}$ activation by a simultaneously stimulated Gs-coupled receptor. ${ }^{90}$ Our hypothesis has been in fact demonstrated for the target of caffeine, the striatal A2AR-D2R heterotetramer. ${ }^{3-5}$

The striatal A2AR-D2R heterotetramer constitutes, therefore, a more real target for the previously demonstrated strong interactions between striatal adenosine and dopamine. ${ }^{4,7}$ The presence of these interactions in 
the dorsal striatum has fostered the rational of applying A2AR antagonists in Parkinson's disease. ${ }^{12,13}$ However, the A2AR-D2R heteromer is also localized in the ventral striatum, involved in more motivational aspects of behavior. $^{3,38}$

As reviewed in this study, this implies to the use of A2AR antagonists and caffeine in other neuropsychiatric disorders, such as depression and ADHD. ${ }^{16,49}$ We also demonstrated that another target of caffeine, the A1RD1R heteromer, also sustains the canonical antagonistic Gi-Gs interaction at the AC level, suggesting a heterotetrameric structure. ${ }^{10}$ Significantly, the A1R-D1R was recently identified in the spinal cord, where it plays an important role in the modulation of the excitability of the spinal MN. ${ }^{10}$ Its pharmacological properties indicate that the spinal A1R-D1R heteromer can constitute a therapeutic target for SCI.

\section{Acknowledgments}

The authors thank their research collaborators, Dr. Merce Correa and Ms. Angela Patricia França, and Dr. Leonardo Pardo for his permission to reproduce his artwork in Figure 1. This work was supported by the intramural funds of the National Institute on Drug Abuse, COBRE Center for Neuroplasticity (NIH NIGMS 1P20GM103642), US NIH/NIMH (MH094966), the UCONN Research Foundation, Shire and Prexa, MEC (PSI2015-68497-R), "Conselho Nacional de Desenvolvimento Científico e Tecnológico" (CNPq, Universal 408676/2016-7), “Coordenação de Aperfeiçoamento de Pessoal de Nível Superior' (CAPES-FCT), and Programa de Apoio aos Núcleos de Excelência (PRONEXProject NENASC).

\section{Author Disclosure Statement}

No competing financial interests exist.

\section{References}

1. Ferré $\mathrm{S}$. Role of the central ascending neurotransmitter systems in the psychostimulant effects of caffeine. J Alzheimers Dis. 2010;20 Suppl 1:S35-S49.

2. Ferré S. Mechanisms of the psychostimulant effects of caffeine: Implications for substance use disorders. Psychopharmacology. 2016;233:1963-1979.

3. Ferré S, Bonaventura J, Zhu W, et al. essential control of the function of the striatopallidal neuron by pre-coupled complexes of adenosine $\mathrm{A}(2 \mathrm{~A})$-dopamine $\mathrm{D}(2)$ receptor heterotetramers and adenylyl cyclase. Front Pharmacol. 2018;9:243.

4. Bonaventura J, Navarro G, Casadó-Anguera V, et al. Allosteric interactions between agonists and antagonists within the adenosine $\mathrm{A} 2 \mathrm{~A}$ receptor-dopamine $\mathrm{D} 2$ receptor heterotetramer. Proc Natl Acad Sci U S A. 2015;112: E3609-E3618.

5. Navarro G, Cordomí A, Casadó-Anguera V, et al. Evidence for functional pre-coupled complexes of receptor heteromers and adenylyl cyclase. Nat Commun. 2018; 9:1242.

6. Orru M, Bakešová J, Brugarolas M, et al. Striatal preand postsynaptic profile of adenosine $\mathrm{A}(2 \mathrm{~A})$ receptor antagonists. PLoS One. 2011;6:e16088.

7. Taura J, Valle-León M, Sahlholm K, et al. Behavioral control by striatal adenosine $\mathrm{A}(2 \mathrm{~A})$-dopamine $\mathrm{D}(2)$ receptor heteromers. Genes Brain Behav. 2018;17:e12432.

8. Ferré S. An update on the mechanisms of the psychostimulant effects of caffeine. J Neurochem. 2008;105: 1067-1079.

9. Ciruela F, Casadó V, Rodrigues RJ, et al. Presynaptic control of striatal glutamatergic neurotransmission by adenosine A1-A2A receptor heteromers. J Neurosci. 2006;26:2080-2087.

10. Rivera-Oliver M, Moreno E, Álvarez-Bagnarol Y, et al. Adenosine $\mathrm{A}(1)$-dopamine $\mathrm{D}(1)$ receptor heteromers control the excitability of the spinal motoneuron. Mol Neurobiol. 2018; DOI: 10.1007/s12035-018-1120-y.

11. Acevedo J, Santana-Almansa A, Matos-Vergara N, Marrero-Cordero LR, Cabezas-Bou E, Díaz-Ríos M. Caffeine stimulates locomotor activity in the mammalian spinal cord via adenosine A1 receptor-dopamine D1 receptor interaction and PKA-dependent mechanisms. Neuropharmacology. 2016;101:490-505.

12. Prediger RD. Effects of caffeine in Parkinson's disease: From neuroprotection to the management of motor and non-motor symptoms. J Alzheimers Dis. 2010;20: S205-S220.

13. Armentero MT, Pinna A, Ferré S, Lanciego JL, Müller $\mathrm{CE}$, Franco R. Past, present and future of A(2A) adenosine receptor antagonists in the therapy of Parkinson's disease. Pharmacol Ther. 2011;132:280-299.

14. Salamone JD, Correa M, Nunes EJ, Randall PA, Pardo M. The behavioral pharmacology of effort-related choice behavior: Dopamine, adenosine and beyond. J Exp Anal Behav. 2012;97:125-146.

15. López-Cruz L, Salamone JD, Correa M. Caffeine and selective adenosine receptor antagonists as new therapeutic tools for the motivational symptoms of depression. Front Pharmacol. 2018;9:526.

16. França AP, Takahashi RN, Cunha RA, Prediger RD. Promises of caffeine in attention-deficit/hyperactivity disorder: From animal models to clinical practice. J Caff Ade Res. 2018;8:132-142.

17. Sadana R, Dascal N, Dessauer CW. N terminus of type 5 adenylyl cyclase scaffolds Gs heterotrimer. Mol Pharmacol. 2009;76:1256-1264.

18. Sadana R, Dessauer CW. Physiological roles for G protein-regulated adenylyl cyclase isoforms: Insights from knockout and overexpression studies. Neurosignals. 2009;17:5-22.

19. Dessauer CW, Tesmer JJ, Sprang SR, Gilman AG. Identification of a Gialpha binding site on type V adenylyl cyclase. J Biol Chem. 1998;273:25831-25839.

20. Cabrera-Vera TM, Vanhauwe J, Thomas TO, et al. Insights into $\mathrm{G}$ protein structure, function, and regulation. Endocr Rev. 2003;24:765-781.

21. Ferré S, von Euler G, Johansson B, Fredholm BB, Fuxe K. Stimulation of high-affinity adenosine A2 receptors decreases the affinity of dopamine D2 receptors in rat striatal membranes. Proc Natl Acad Sci U S A. 1991; $88: 7238-7241$. 
22. Ferré S, Fredholm BB, Morelli M, Popoli P, Fuxe K. Adenosine-dopamine receptor-receptor interactions as an integrative mechanism in the basal ganglia. Trends Neurosci. 1997;20:482-487.

23. Azdad K, Gall D, Woods AS, Ledent C, Ferré S, Schiffmann SN. Dopamine D2 and adenosine A2A receptors regulate NMDA-mediated excitation in accumbens neurons through A2A-D2 receptor heteromerization. Neuropsychopharmacology. 2009;34:972-986.

24. Dale N, Gilday D. Regulation of rhythmic movements by purinergic neurotransmitters in frog embryos. Nature. 1996;383:259-263.

25. Witts EC, Panetta KM, Miles GB. Glial-derived adenosine modulates spinal motor networks in mice. J Neurophysiol. 2012;107:1925-1934.

26. Witts EC, Nascimento F, Miles GB. Adenosinemediated modulation of ventral horn interneurons and spinal motoneurons in neonatal mice. J Neurophysiol. 2015;114:2305-2315.

27. Kjaerulff O, Kiehn O. Distribution of networks generating and coordinating locomotor activity in the neonatal rat spinal cord in vitro: A lesion study. J Neurosci. 1996;16:5777-5794.

28. Raastad M, Johnson BR, Kiehn O. The number of postsynaptic currents necessary to produce locomotor-related cyclic information in neurons in the neonatal rat spinal cord. Neuron. 1996;17:729-738.

29. Tresch MC, Kiehn O. Coding of locomotor phase in populations of neurons in rostral and caudal segments of the neonatal rat lumbar spinal cord. J Neurophysiol. 1999; 82:3563-3574.

30. Kiehn O, Butt SJ. Physiological, anatomical and genetic identification of CPG neurons in the developing mammalian spinal cord. Prog Neurobiol. 2003;70:347-361.

31. Clemens S, Belin-Rauscent A, Simmers J, Combes D. Opposing modulatory effects of D1- and D2-like receptor activation on a spinal central pattern generator. J Neurophysiol. 20122;107:2250-2259.

32. Sharples SA, Koblinger K, Humphreys JM, Whelan PJ. Dopamine: A parallel pathway for the modulation of spinal locomotor networks. Front Neural Circuits. 2014;8: 55.

33. Zhu H, Clemens S, Sawchuk M, Hochman S. Expression and distribution of all dopamine receptor subtypes (D(1)$\mathrm{D}(5))$ in the mouse lumbar spinal cord: A real-time polymerase chain reaction and non-autoradiographic in situ hybridization study. Neuroscience. 2007;149:885-897.

34. Miyazaki N, Nakatsuka T, Takeda D, Nohda K, Inoue K, Yoshida M. Adenosine modulates excitatory synaptic transmission and suppresses neuronal death induced by ischaemia in rat spinal motoneurones. Pflugers Arch. 2008;457:441-451.

35. Yang CC, Jou IM. Caffeine treatment aggravates secondary degeneration after spinal cord injury. Brain Res. 2016;1634:75-82.

36. Lapointe NP, Rouleau P, Ung RV, Guertin PA. Specific role of dopamine $\mathrm{D} 1$ receptors in spinal network activation and rhythmic movement induction in vertebrates. J Physiol. 2009;587:1499-1511.

37. Ferré S, Casadó V, Devi LA, et al. G protein-coupled receptor oligomerization revisited: Functional and pharmacological perspectives. Pharmacol Rev. 2014;66: 413-434.
38. Salamone JD, Yohn S, Lopez-Cruz L, San Miguel N, Correa M. Activational and effort-related aspects of motivation: Neural mechanisms and implications for psychopathology. Brain. 2016;139:1325-1347.

39. Fava M, Ball S, Nelson JC, et al. Clinical relevance of fatigue as a residual symptom in major depressive disorder. Depress Anxiety. 2014;31:250-257.

40. Nunes EJ, Randall PA, Santerre JL, et al. Differential effects of selective adenosine antagonists on the effortrelated impairments induced by (DA) D1 and D2 antagonism. Neuroscience. 2010;170:268-280.

41. Nunes EJ, Randall PA, Hart EE, et al. Effort-related motivational effects of the VMAT-2 inhibitor tetrabenazine: Implications for animal models of the motivational symptoms of depression. J Neurosci. 2013;33:19120 19130.

42. Randall PA, Pardo M, Nunes EJ, et al. Dopaminergic modulation of effort-related choice behavior as assessed by a progressive ratio chow task: Pharmacological studies and role of individual differences. PLoS One. 2012;7: e47934.

43. Randall PA, Lee CA, Nunes EJ, et al. The VMAT-2 inhibitor tetrabenazine affects effort-related decision making in a progressive ratio/chow feeding choice task: Reversal with antidepressant drugs. PLoS One. 2014;9: e99320.

44. Yohn SE, Thompson C, Randall PA, et al. The VMAT-2 inhibitor tetrabenazine alters effort-related decision making as measured by the T-maze barrier choice task: Reversal with the adenosine A2A antagonist MSX-3 and the catecholamine uptake blocker bupropion. Psychopharmacology. 2015;232:1313-1323.

45. Yohn SE, Collins SL, Contreras-Mora HM, et al. Not all antidepressants are created equal: Differential effects of monoamine uptake inhibitors on effort-related choice behavior. Neuropsychopharmacology. 2016;41:686-694.

46. Yohn SE, Lopez-Cruz L, Hutson PH, Correa M, Salamone JD. Effects of lisdexamfetamine and s-citalopram, alone and in combination, on effort-related choice behavior in the rat. Psychopharmacology. 2016;233:949-960.

47. Yohn SE, Gogoj A, Haque A, et al. Evaluation of the effort-related motivational effects of the novel dopamine uptake inhibitor PRX-14040. Pharmacol Biochem Behav. 2016;148:84-91.

48. Bryce CA, Floresco SB. Perturbations in effort-related decision-making driven by acute stress and corticotropinreleasing factor. Neuropsychopharmacology. 2016;41: 2147-2159.

49. Salamone JD, Correa M, Ferrigno S, Yang JH, Rotolo RA, Presby RE. The psychopharmacology of effortrelated decision making: Dopamine, adenosine, and insights into the neurochemistry of motivation. Pharmacol Rev. 2018;70:747-762.

50. Nunes EJ, Randall PA, Estrada A, et al. Effort-related motivational effects of the pro-inflammatory cytokine interleukin 1-beta: Studies with the concurrent fixed ratio 5/chow feeding choice task. Psychopharmacology. 2014;231:727-736.

51. Yohn SE, Arif Y, Haley A, et al. Effort-related motivational effects of the pro-inflammatory cytokine interleukin-6: Pharmacological and neurochemical characterization. Psychopharmacology. 2016;233:35753586. 
52. Treadway MT, Zald DH. Parsing anhedonia: translational models of reward-processing deficits in psychopathology. Curr Dir Psychol Sci. 2013;22:244-249.

53. Yang XH, Huang J, Zhu CY, et al. Motivational deficits in effort-based decision making in individuals with subsyndromal depression, first-episode and remitted depression patients. Psychiatry Res. 2014;220:874-882.

54. Mingote S, Font L, Farrar AM, et al. Nucleus accumbens adenosine A2A receptors regulate exertion of effort by acting on the ventral striatopallidal pathway. J Neurosci. 2008;28:9037-9046.

55. Farrar AM, Pereira M, Velasco F, Hockemeyer J, Muller $\mathrm{CE}$, Salamone JD. Adenosine A(2A) receptor antagonism reverses the effects of (DA) receptor antagonism on instrumental output and effort-related choice in the rat: Implications for studies of psychomotor slowing. Psychopharmacology. 2007;191:579-586.

56. Salamone JD, Farrar AM, Font L, et al. Differential actions of adenosine $\mathrm{A} 1$ and $\mathrm{A} 2 \mathrm{~A}$ antagonists on the effortrelated effects of (DA) D2 antagonism. Behav Brain Res. 2010;201:216-222.

57. Mott AM, Nunes EJ, Collins LE, et al. The adenosine A2A antagonist MSX-3 reverses the effects of the dopamine antagonist haloperidol on effort-related decision making in a T-maze cost/benefit procedure. Psychopharmacology. 2009;204:103-112.

58. Worden LT, Shahriari M, Farrar AM, et al. The adenosine A2A antagonist MSX-3 reverses the effort-related effects of dopamine blockade: Differential interaction with D1 and D2 family antagonists. Psychopharmacology. 2009;203:489-499.

59. Santerre JL, Nunes EJ, Randall PA, Baqi Y, Müller CE, Salamone JD. Behavioral studies with the novel adenosine A2A antagonist MSX-4: Reversal of the effects of (DA) D2 antagonism. Pharmacol Biochem Behav. 2012; 102:477-487.

60. Pardo M, Lopez-Cruz L. Valverde O, et al. Adenosine A2A receptor antagonism and genetic deletion attenuate the effects of dopamine D2 antagonism on effort-based decision making in mice. Neuropharmacology. 2012; 62:2068-2077.

61. Nomoto M, Nagai M, Nishikawa N. Clinical nonmotor aspect of A2A antagonist in PD treatment. Int Rev Neurobiol. 2014;119:191-194.

62. Abe K, Fujita M, Yoshikawa H. Effectiveness of istradefylline for fatigue and quality of life in Parkinson's disease patients' and of their caregivers'. Adv Parkinsons Dis. 2016;5:24-28.

63. SanMiguel N, Pardo M, Carratalá-Ros C, López-Cruz L, Salamone JD, Correa M. Individual differences in the energizing effects of caffeine on effort-based decisionmaking tests in rats. Pharmacol Biochem Behav. 2018; 169:27-34.

64. Polanczyk GV, Willcutt EG, Salum GA, Kieling C, Rohde LA. ADHD prevalence estimates across three decades: An updated systematic review and meta-regression analysis. Int J Epidemiol. 2014;43:434-442.

65. Moffitt TE, Houts R, Asherson P, et al. Is adult ADHD a childhood-onset neurodevelopmental disorder? Evidence from a four-decade longitudinal cohort study. Am J Psychiatry. 2015;172:967-977.

66. Swanson JM, Kinsbourne M, Nigg J, et al. Etiologic subtypes of attention- deficit/hyperactivity disorder: Brain imaging, molecular genetic and environmental factors and the dopamine hypothesis. Neuropsychol Rev. 2007;17:39-59.

67. Catalá-López F, Hutton B, Núñez-Beltrán A, et al. The pharmacological and non-pharmacological treatment of attention deficit hyperactivity disorder in children and adolescents: A systematic review with network metaanalyses of randomised trials. PLoS One. 2017;12: $\mathrm{e} 0180355$.

68. Takahashi RN, Pamplona FA, Prediger RD. Adenosine receptor antagonists for cognitive dysfunction: A review of animal studies. Front Biosci. 2008;13:2614-2632.

69. Cunha RA, Ferré S, Vaugeois JM, Chen JF. Potential therapeutic interest of adenosine A2A receptors in psychiatric disorders. Curr Pharm Des. 2008;14:1512-1524.

70. Schnackenberg RC. Caffeine as a substitute for schedule II stimulants in hyperkinetic children. Am J Psychiatry. 1973;130:796-798.

71. Reichard CC, Elder ST. The effects of caffeine on reaction time in hyperkinetic and normal children. Am J Psychiatry. 1977;134:144-148.

72. Kupietz SS, Winsberg BG. Caffeine and inattentiveness in reading-disabled children. Percept Mot Skills. 1977; 44:1238.

73. Harvey DH, Marsh RW. The effects of de-caffeinated coffee versus whole coffee on hyperactive children. Dev Med Child Neurol. 1978;20:81-86.

74. Firestone P, Poitras-Wright H, Douglas V. The effects of caffeine on hyperactive children. J Learn Disabil. 1978; 11:133-141.

75. Conners CK. A placebo-crossover study of caffeine treatment of hyperkinetic children. Int J Ment Health. 1975;4:132-143.

76. Gross MD. Caffeine in the treatment of children with minimal brain dysfunction or hyperkinetic syndrome. Psychosomatics. 1975;16:26-27.

77. Huestis RD, Arnold LE, Smeltzer DJ. Caffeine versus methylphenidate and d-amphetamine in minimal brain dysfunction: A double-blind comparison. Am J Psychiatry. 1975;132:868-870.

78. Arnold LE, Christopher J, Huestis R, Smeltzer DJ. Methylphenidate vs dextroamphetamine vs caffeine in minimal brain dysfunction: Controlled comparison by placebo washout design with Bayes' analysis. Arch Gen Psychiatry. 1978;35:463-473.

79. Ioannidis K, Chamberlain SR, Muller U. Ostracising caffeine from the pharmacological arsenal for attention-deficit hyperactivity disorder-Was this a correct decision? A literature review. J Psychopharmacol. 2014;28:830-836.

80. Leon MR. Effects of caffeine on cognitive, psychomotor, and affective performance of children with attention-deficit/hyperactivity disorder. J Atten Disord. 2000;4:27-47.

81. Cornelis MC, Kacprowski T, Menni C, et al. Genomewide association study of caffeine metabolites provides new insights to caffeine metabolism and dietary caffeineconsumption behavior. Hum Mol Genet. 2016;25:54725482.

82. Prediger RD, Fernandes D, Takahashi RN. Blockade of adenosine A2A receptors reverses short-term social memory impairments in spontaneously hypertensive rats. Behav Brain Res. 2005; 159:197-205.

83. Pires VA, Pamplona FA, Pandolfo P, Fernandes D, Prediger RD, Takahashi RN. Adenosine receptor antagonists 
improve short-term object-recognition ability of spontaneously hypertensive rats: A rodent model of attentiondeficit hyperactivity disorder. Behav Pharmacol. 2009; 20:134-145.

84. Prediger RD, Pamplona FA, Fernandes D, Takahashi RN. Caffeine improves spatial learning deficits in an animal model of attention deficit hyperactivity disorder (ADHD) - The spontaneously hypertensive rat (SHR). Int J Neuropsychopharmacol. 2005;8:583-594.

85. Pires VA, Pamplona FA, Pandolfo P, Prediger RD, Takahashi RN. Chronic caffeine treatment during prepubertal period confers long-term cognitive benefits in adult spontaneously hypertensive rats (SHR), an animal model of attention deficit hyperactivity disorder (ADHD). Behav Brain Res. 2010;215:39-44.

86. Pandolfo P, Machado NJ, Kofalvi A, Takahashi RN, Cunha RA. Caffeine regulates frontocorticostriatal dopamine transporter density and improves attention and cognitive deficits in an animal model of attention deficit hyperactivity disorder. Eur Neuropsychopharmacol. 2013;23:317-328.

87. Nunes F, Pochmann D, Almeida AS, Marques DM, Porciuncula LO. Differential behavioral and biochemical re- sponses to caffeine in male and female rats from a validated model of attention deficit and hyperactivity disorder. Mol Neurobiol. 2018;55:8486-8498.

88. Ribeiro FF, Xapelli S, Miranda-Lourenço C, et al. Purine nucleosides in neuroregeneration and neuroprotection. Neuropharmacology. 2016;104:226-242.

89. Ferré S, Baler R, Bouvier M, et al. Building a new conceptual framework for receptor heteromers. Nat Chem Biol. 2009;5:131-134.

90. Ferré S. The GPCR heterotetramer: Challenging classical pharmacology. Trends Pharmacol Sci. 2015;36: $145-152$.

Address correspondence to: Sergi Ferré, $M D, P h D$ Integrative Neurobiology Section Intramural Research Program National Institute on Drug Abuse National Institutes of Health Triad Technology Building, 333 Cassell Drive Baltimore, MD 21224

E-mail: sferre@intra.nida.nih.gov 